\title{
Comment and Response in Attention, Perception, \& Psychophysics
}

In this issue, readers will find an exchange of views on the topic of visual neglect between Shomstein, Kimchi, Hammer, and Behrmann, on the one hand, and Milberg and McGlinchey, on the other (pp. 607-627). In this case, the exchange grew out of the review process. Sometimes, reviewers and authors simply disagree on a point. In most cases, these are matters for the paper's action editor to sort out. In rare cases, the issue seems interesting enough to present to the readership. The question of the presence or absence of attentional selection in neglect seemed to me to be one of those issues, so I invited Bill Milberg to write a comment and Sarah Shomstein and her coauthors to write a response. I hope readers will find the exchange interesting, but I am highlighting it here in an editorial in order to make a few broader points.

1. It is not necessary for all reviewers to agree with everything an author says in order for a paper to be published in $A P \& P$.

2. The manuscript submission process allows authors to recommend reviewers and to ask that certain people not serve as reviewers. As a corollary of Point 1, it is usually not necessary to keep one's ideological opponents away from the review process. They are likely to be among the most interested readers of an author's paper. Authors should feel free to use their cover letter to tell us that Dr. X is likely to be unhappy with the findings but, assuming that those findings are solid, why not use the review process to gently coerce Dr. X into engaging with one's work? Of course, there are cases where Dr. X tries to use the review process as a mechanism to block publication of contrary views. This is not very common; at least, not in the reviews that I see.

3. Exchanges such as the one published this month need not arise exclusively out of the review process. If you believe that some paper in $A P \& P$ calls for a comment, please send me a short e-mail atwolfe@ssearch.bwh.harvard.edu. Describe the main point of the comment and tell us why this is important. $A P \& P$ is not a commentary journal, so the bar for submissions of this sort is very high. In particular, this is not the venue to narrowly defend the conclusions of one's last three papers. However, if you think that a paper raises a fundamental issue of broad interest that should be discussed, feel free to suggest it. If we decide to move forward, we would always invite the authors of the target article to respond, and both the comment and the response would be subject to peer review.

Jeremy M. Wolfe Editor 